\title{
Is optical Fe II emission related to the soft X-ray properties of quasars?
}

\section{Citation}

Wilkes, Belinda J., Martin Elvis, and lan McHardy. 1987. “Is Optical Fe II Emission Related to the Soft X-Ray Properties of Quasars?" The Astrophysical Journal 321 (October): L23. doi:10.1086/184999.

\section{Published Version}

10.1086/184999

\section{Permanent link}

http://nrs.harvard.edu/urn-3:HUL.InstRepos:30248673

\section{Terms of Use}

This article was downloaded from Harvard University's DASH repository, and is made available under the terms and conditions applicable to Other Posted Material, as set forth at http:// nrs.harvard.edu/urn-3:HUL.InstRepos:dash.current.terms-of-use\#LAA

\section{Share Your Story}

The Harvard community has made this article openly available.

Please share how this access benefits you. Submit a story.

\section{Accessibility}


The ASTROPHYSICAL JouRnal, 321:L23-L27, 1987 October 1

(C) 1987. The American Astronomical Society. All rights reserved. Printed in U.S.A.

\title{
IS OPTICAL Fe II EMISSION RELATED TO THE SOFT X-RAY PROPERTIES OF QUASARS?
}

\author{
Belinda J. Wilkes AND Martin ElVis \\ Harvard-Smithsonian Center for Astrophysics \\ AND \\ IAN MCHARDY \\ X-Ray Astronomy Group, University of Leicester \\ Received 1987 March 13; accepted 1987 July 22
}

\begin{abstract}
Radio-quiet quasars generally show broad, blended multiplets of $\mathrm{Fe}$ II emission in their optical and ultraviolet (UV) spectra. Radio-loud quasars also show UV Fe II emission, but their optical $\mathrm{Fe}$ II emission is generally weaker. No satisfactory theory connecting the generation of Fe II and radio emission has been found to explain this effect. A second, well-established distinction between the two classes of quasar is in their X-ray properties: radio-loud quasars are more $\mathrm{X}$-ray luminous, and recent results have shown that they also have systematically flatter soft X-ray slopes. We propose that the second effect causes the first, i.e., that the primary factor controlling the optical Fe II emission is the soft X-ray spectrum. This proposition is supported by X-ray and optical data for nine quasars which shows a correlation between the soft X-ray slope and the strength of the optical Fe II emission. One of these quasars, $1803+676$, is radio-quiet and yet its optical spectrum shows no evidence for $\mathrm{Fe}$ II emission. This quasar is also unusual in that it has a flat X-ray spectrum. This further supports our proposal that the X-ray spectrum is important in determining the relative strengths of UV and optical Fe II emission.
\end{abstract}

Subject headings: quasars - X-rays: spectra

\section{INTRODUCTION}

The prominent optical and ultraviolet (UV) emission lines in quasar spectra are generally believed to be produced by photoionization of the emitting gas by the "nonthermal" UV to X-ray continuum (see Ferland and Shields 1985 for a recent review). The observed emission line ratios vary by quite large factors $(\sim 5)$ from object to object. Photoionization models predict that these ratios depend primarily upon the shape of the ionizing continuum and the ionization parameter. There have been very limited studies investigating the relation between the shape of the ionizing continuum and emission-line strengths. This is because the extreme UV, from the Lyman limit to $\sim 100 \AA$, is essentially unobservable due to interstellar absorption and spectral information on the soft $\mathrm{X}$-ray continuum has only recently become available with the calibration of the Imaging Proportional Counter (IPC) on the Einstein Observatory and with EXOSAT. This Letter takes advantage of these new data.

The strongest known connection between the emission-line properties of quasars is not (apparently) related to the ionizing continuum. Radio-quiet quasars possess many strong, heavily blended, optical and UV Fe II [Fe II(UV)] emission lines. In radio-loud quasars $\mathrm{Fe} \mathrm{II}(\mathrm{UV})$ emission is present, but optical Fe II [Fe II(opt)] is generally weak or absent (Peterson, Foltz, and Byard 1981; Phillips 1977; Osterbrock 1977). The observed strength of emission in the Fe II blends (both UV and optical) is often large, comparable with that of Ly $\alpha$
1215. A variety of mechanisms for generating this $\mathrm{Fe}$ II emission have been considered, but none of them predicts the observed link between $\mathrm{Fe} \mathrm{II}(\mathrm{opt})$ and radio emission.

Radio-loud and radio-quiet quasars also differ in their $\mathrm{X}$-ray properties. Radio-loud quasars are more luminous X-ray sources than radio-quiet quasars (see, e.g., Zamorani et al. 1981; Kembhavi, Feigelson, and Singh 1985). This effect is generally interpreted as being due to an additional X-ray component directly linked to the presence of radio emission, possibly via the synchrotron self-Compton process. Wilkes and Elvis (1987) have recently shown that, in addition, the soft X-ray slopes $(0.1-3.5 \mathrm{keV})$ of radio-loud quasars are flatter than those of radio-quiet quasars. Given that radioloudness is related to both the Fe II(opt) strength and the soft $\mathrm{X}$-ray slope, we would expect that the two latter quantities are also related. We investigate this possibility here.

Although Fe II(opt) measurements exist in the literature for a number of quasars in the Wilkes and Elvis sample, the combination of the nonuniformity of these measurements; the different possible measurement procedures, which are rarely described; and the difficulty with finding an appropriate continuum level among the highly blended Fe II(opt) emission lines, as described by Wills (1987); conspire to make these data of dubious value for further testing the effect. We have instead measured the Fe II(opt) emission for those few quasars whose optical spectra are available to us, thus ensuring uniformity of measurement. 


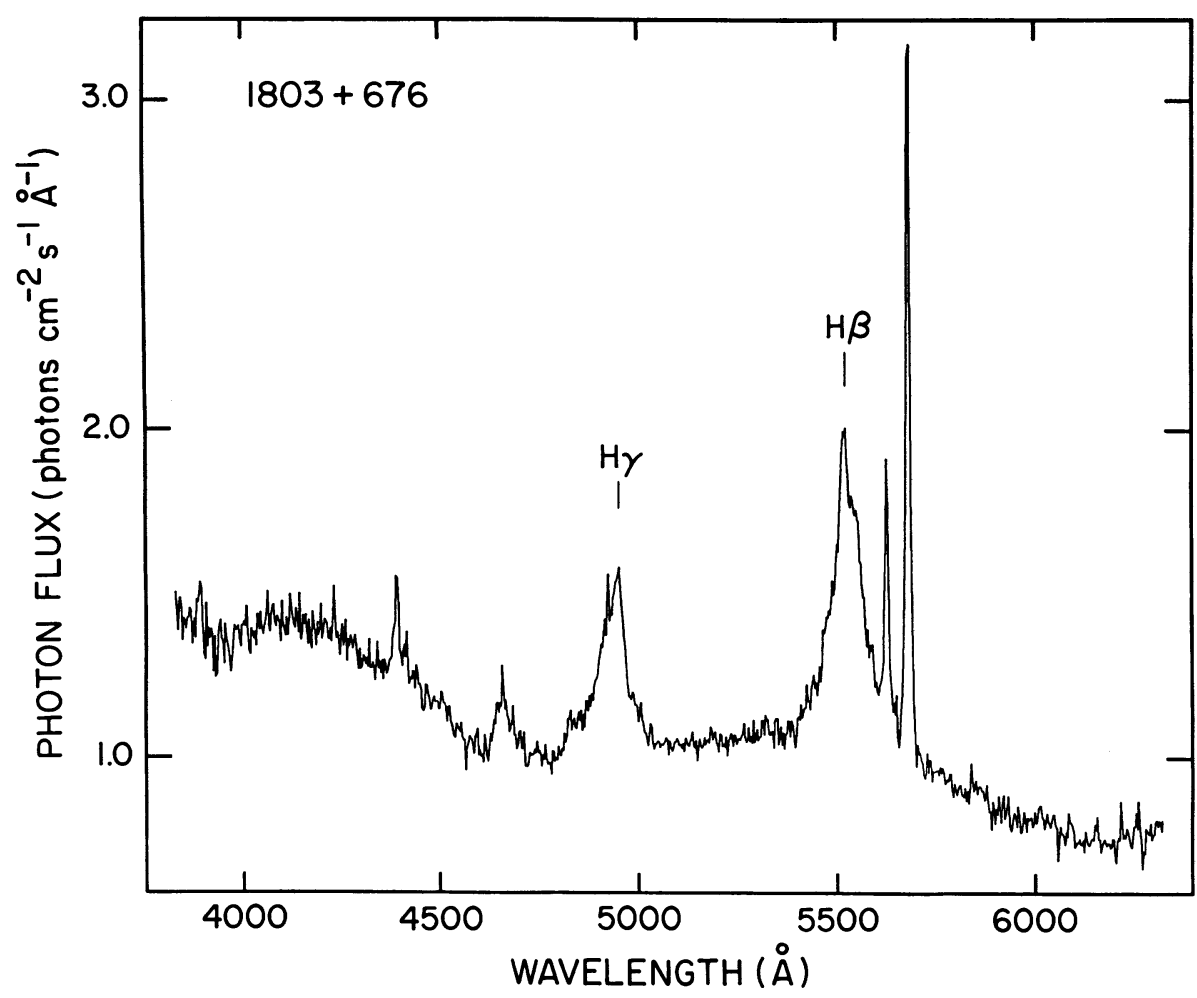

FIG. 1. - MMT spectrum of $1803+676$ showing lack of obvious Fe II emission between $\mathrm{H} \beta$ and $\mathrm{H} \gamma$ and longward of $\mathrm{H} \beta$ ( $z=0.136)$

\section{OPTICAL DATA}

Optical spectra for four of the Wilkes and Elvis quasars were obtained with the $4 \mathrm{~m}$ Anglo-Australian Telescope as part of a program to obtain redshifts of Parkes flat-spectrum radio sources (Wilkes et al. 1983; Peterson et al. 1979 and references therein). The $\mathrm{Fe}$ II $\lambda 4570$ and $\mathrm{H} \beta \lambda 4861$ features $^{1}$ in these spectra were measured by fitting a power-law continuum through $\sim 20-30 \AA$ regions on either side of the feature; for Fe II: $4450 \AA$ and $4740 \AA$; for $\mathrm{H} \beta$ : $4740 \AA$ and $5060 \AA$. ([O III] $\lambda \lambda 4959,5007$ were first removed from the red wing of $H \beta$.) $1803+676$, also in the Wilkes and Elvis sample, was observed on the Multiple Mirror Telescope (MMT) with the MMT spectrograph and 300 lines $\mathrm{mm}^{-1}$ grating in 1982 September. A high signal-to-noise ratio $(S / N)$ observation was made through $1^{\prime \prime} \times 2$ "' 6 rectangular apertures giving $\sim 5$ $\AA$ resolution. A second spectrum was obtained through 5" circular apertures to minimize the loss of blue light due to atmospheric differential refraction. The latter spectrum was flux calibrated with reference to the standard star EG 129 (Oke 1974) observed immediately beforehand using the same spectrograph setup. The high $S / N\left(1^{\prime \prime} \times 22^{\prime \prime} 6\right.$ aperture $)$ spectrum, normalized to the continuum of the calibrated spectrum, is shown in Figure 1. This spectrum and that of PG $1211+143$, reported in Bechtold et al. (1987), were also measured as described above. In addition, Kriss, McHardy, and Malkan (1987) present soft X-ray slopes and optical spectra

\footnotetext{
${ }^{1} \mathrm{H} \beta$ measurements are reported in Wilkes (1986). The line was remeasured here to ensure consistency with the other quasars in this paper. Differences between these and the earlier measurements are within the expected errors ( 10\% for FWHM).
}

for three additional radio-quiet quasars. The X-ray data are derived from EXOSAT LE (low-energy) filter data and a single EXOSAT ME (medium-energy) data point at $\sim 3$ keV. (Technical details of EXOSAT are described in Taylor et al. 1981.) Their energy range is thus quite comparable to the IPC. Fe II $\lambda 4570$ and $\mathrm{H} \beta$ strengths and widths have been extracted from the Kriss et al. optical spectra using the procedure described above. All the quasars have $\mathrm{H} \beta$ widths $<5000$ $\mathrm{km} \mathrm{s}^{-1}$. Based upon the results of Wills (1987), we do not expect line blending in the $\mathrm{Fe} \mathbf{I I}(\mathrm{opt})$ multiplets to significantly affect the measured $\mathrm{Fe}$ II strength in these objects, although we note that our method of measurement is different. For all nine quasars the $\mathrm{X}$-ray slopes, the observed equivalent widths of $\mathrm{Fe}$ II $\lambda 4570$, its strength relative to $\mathrm{H} \beta$ $\lambda 4861$ and the observed full width at half-maximum (FWHM) of $\mathrm{H} \beta$ are given in Table 1 in order of increasing X-ray slope. In Figure 2 the $\mathrm{Fe}$ il equivalent widths are plotted as a function of the soft X-ray slope. The quasar redshifts are low $(\lessgtr 0.3)$ so that conversion to rest frame values is unnecessary.

\section{DISCUSSION}

Although this is a crude measurement of $\mathrm{Fe} \mathrm{II(opt),} \mathrm{with} \mathrm{no}$ allowance made for the presence of He II $\lambda 4648$ emission or differing emission line widths, there is a clear trend of increasing $\mathrm{Fe} \mathrm{II}(\mathrm{opt})$ strength with steepening of the soft X-ray slope (Fig. 2). The correlation coefficient is 0.894 (99.8\% significance). We note that Remillard and Schwartz $(1987 a, b)$ have found a similar relation from optical spectroscopy and EXOSAT spectra of hard X-ray selected quasars from the HEAO 1 A-3 survey. 
No. 1,1987

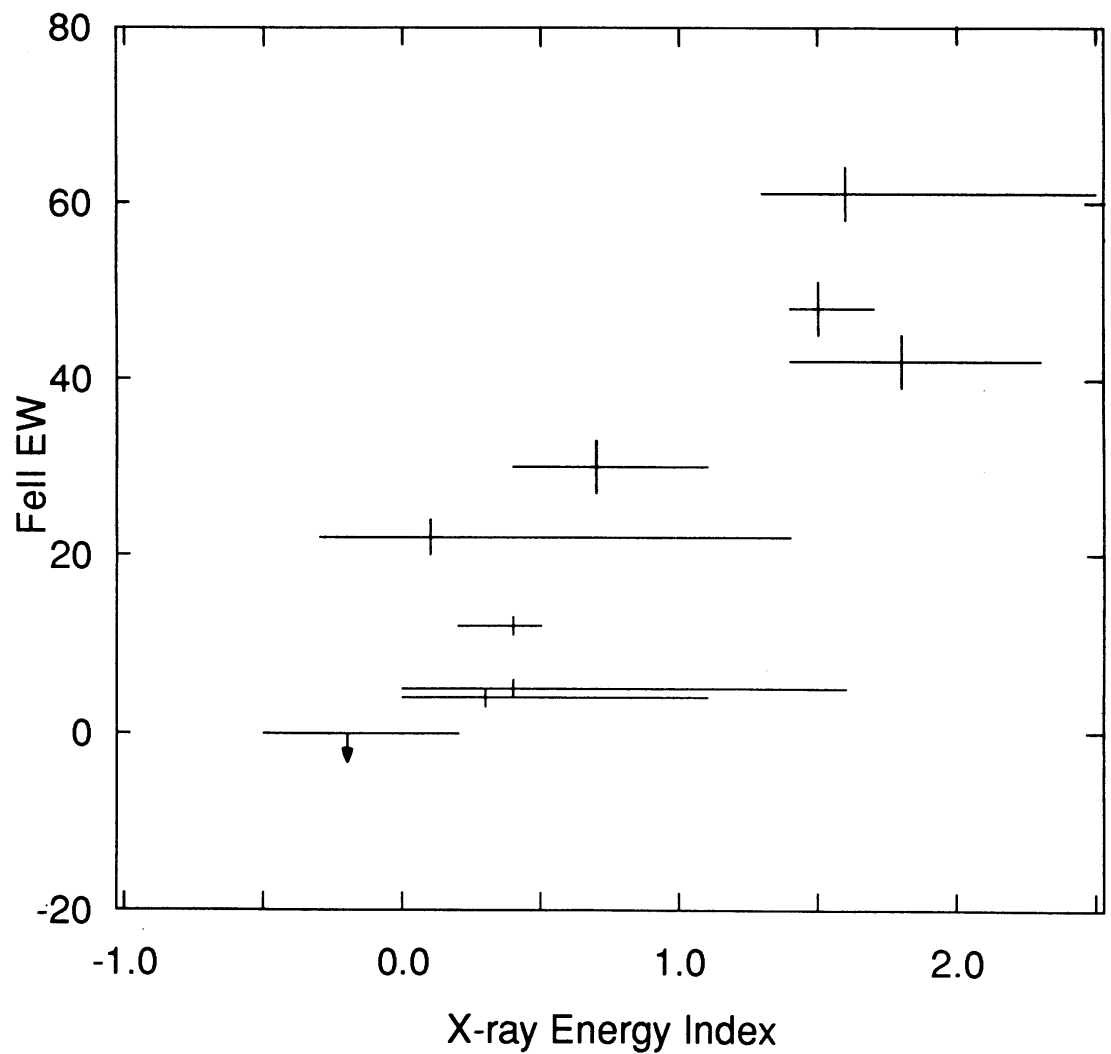

FIg. 2. - Observed equivalent width (in $\AA$ ) of the Fe II $\lambda 4570$ blend as a function of soft X-ray slope. EW width errors are estimated at $\sim 10 \%$; X-ray errors are $90 \%$ confidence limits for two parameters.

TABLE 1

X-Ray Slopes and Optical Fe II Strengths ${ }^{\text {a }}$ of Quasar

\begin{tabular}{|c|c|c|c|c|}
\hline Quasar & X-Ray Energy Index ${ }^{b}$ & $\mathrm{Fe}$ II EW( $(\AA)^{\mathrm{c}}$ & Fe II:H $\beta$ & $\overline{\mathrm{H} \beta: \mathrm{FWHM}^{\mathrm{d}}}$ \\
\hline $1803+676 \ldots \ldots \ldots$ & $-0.2_{-0.3}^{+0.4}$ & $<0.2$ & $<0.005$ & 3800 \\
\hline $0312-770 \ldots \ldots \ldots$ & $0.1_{-0.4}^{+1.3}$ & 22 & 0.21 & 3340 \\
\hline $1146-037 \ldots \ldots \ldots$ & $0.3_{-0.3}^{+0.8}$ & 4 & 0.05 & 4300 \\
\hline III Zw $2 \ldots \ldots \ldots$ & $0.4_{-0.4}^{+1.2}$ & 5 & 0.06 & 4700 \\
\hline 3C $273 \ldots \ldots \ldots \ldots$ & $0.4_{-0.2}^{+0.1}$ & 12 & 0.2 & 4200 \\
\hline PG $0923+129 \ldots$ & $0.7_{-0.3}^{+0.4}$ & 30 & 0.69 & 1840 \\
\hline PG $1229+204 \ldots \ldots$ & $1.5_{-0.1}^{+0.2}$ & 48 & 0.67 & 3170 \\
\hline PG $0844+349 \ldots \ldots$ & $1.6_{-0.3}^{+0.9}$ & 61 & 0.97 & 2480 \\
\hline PG $1211+143 \ldots \ldots$ & $1.8_{-0.4}^{+0.5}$ & 42 & 0.65 & 1620 \\
\hline
\end{tabular}

${ }^{a}$ Measured between $\mathrm{H} \beta$ and $\mathrm{H} \gamma$, as described in the text.

b $90 \%$, two-parameter error, from Wilkes and Elvis 1987 or Kriss et al. 1987.

${ }^{\mathrm{c}}$ In observed frame.

${ }^{\mathrm{d}} \mathrm{km} \mathrm{s}^{-1}$ in observed frame.

Since radio-loudness and X-ray slope are well correlated, it is intrinsically difficult to determine which quantity is fundamentally related to the optical Fe II(opt) emission. The nine crude measurements presented here are insufficient and there is no sample of quasars available for which both X-ray and careful, systematic $\mathrm{Fe}$ II(opt) measurements have been made which would allow a principle component analysis. A clear distinction could be made, for individual quasars at least, with $\mathrm{Fe} \mathrm{II}(\mathrm{opt})$ measurements for either radio-loud quasars with steep soft $\mathrm{X}$-ray slopes or conversely radio-quiet quasars with flat soft X-ray slopes. There is one such crucial object in the Wilkes and Elvis (1987) sample: $1803+676$ (KAZ 102; Kazaryan, Carswell, and Khachikyan 1974).

$1803+676$ is a radio-quiet quasar (Hutchings and Gower $1985)$, yet it has a best fit soft X-ray slope, $\alpha_{E}$, of -0.2 $\left(F_{\nu} \propto \nu^{-\alpha_{E}}\right)$, flatter than the flattest radio-loud quasar in the Wilkes and Elvis sample. If weak radio-emission implies strong Fe II(opt) emission then $1803+676$ should show strong $\mathrm{Fe}$ II(opt). If instead flat $\mathrm{X}$-ray slopes are associated with weaker $\mathrm{Fe} \mathrm{II}(\mathrm{opt})$ lines then these lines should be weak in 
$1803+676$. Our spectrum (Fig. 1) shows no apparent Fe II(opt) emission. In particular Fe II $\lambda 4570$, which generally appears as a broad feature between $\mathrm{H} \beta \lambda 4861$ and $\mathrm{H} \gamma \lambda 4340$, is not seen (e.g., compare with spectra in Peterson, Foltz, and Byard 1981). Fe II $\lambda \lambda 4924,5018$ are also not apparent, and a possible feature at Fe II $\lambda 5169$ is very weak. While we do not claim high photometric accuracy for the spectrum in Figure 1, it is sufficient to show that the excess emission at the blue end is consistent with Balmer continuum emission, generally a broad ( $\sim 800 \AA$ ) feature spanning the Balmer limit at $3647 \AA$. The continuum drops considerably longward of $\mathrm{H} \beta$; while this is most likely due to poor flux calibration, we cannot rule out the possibility that $\mathrm{Fe}$ II emission is present between $\mathrm{H} \beta$ and $\mathrm{H} \gamma$ at exactly the right level to produce a smooth flat continuum at a higher level. However, this seems rather contrived particularly since the associated $\mathrm{Fe}$ II emission lines longward of [O III] are absent. We thus conclude that $1803+$ 676 has weak or no Fe II(opt) emission. As noted above this is unusual for a radio-quiet quasar and suggests that the soft $\mathrm{X}$-ray slope is more important than the radio emission in determining the strength of the optical Fe II(opt) emission.

Theoretical arguments also lead us in this direction. The emitting clouds in the broad emission line region (BELR) of quasars are generally believed to be photoionized by continuum emission from a powerful, nonthermal central source. The shape and normalization of the incident $\mathrm{X}$-ray spectrum is crucial in determining the ionization balance in these clouds (Netzer 1987). With the discovery of strong ultraviolet Fe II emission lines, collisional excitation was concluded to dominate the $\mathrm{Fe}$ II line production (Wills et al. 1980; CollinSouffrin et al. 1980; Kwan and Krolik 1981). X-rays penetrate deep into the clouds creating a warm, partially ionized zone at high optical depths where low ionization lines, such as Fe II emission, are generated (Kwan and Krolik 1981). The ratio $\mathrm{Fe}$ II(opt)/Fe II(UV) increases with the optical depth in the Fe II(UV) resonance lines (Netzer and Wills 1983). Thus both the total strength of the $\mathrm{Fe}$ II emission and the ratio $\mathrm{Fe} \mathrm{II}(\mathrm{UV}) / \mathrm{Fe} \mathrm{II}(\mathrm{opt})$ are expected to be strongly related to the X-ray flux incident upon the BELR clouds. On the other hand, no obvious link is expected between Fe II(opt) and a quasar's radio emission.

Based upon our observational results and these theoretical considerations, we suggest that the Fe II(opt) strength is fundamentally related to the slope in the soft X-ray region $(0.1-3.5 \mathrm{keV})$. The $\mathrm{Fe} \mathrm{II}(\mathrm{opt}) /$ radio-loudness relation is then a secondary relation resulting from the primary relation between the soft $\mathrm{X}$-ray slope and radio-loudness.

It should be noted that, while an X-ray/Fe II(opt) relation is expected, the sense of the relation predicted by most current photoionization models of the emitting gas is opposite to that we observe (Krolik 1987). In these models Fe II does not become a dominant coolant until high column densities $10^{22}-10^{23}$ atoms $\mathrm{cm}^{-3}$; in this region the gas is heated mainly by photons at $\geq 1 \mathrm{keV}$. Since the more efficient soft photons are then absent, the overall Fe II emission will be higher for those quasars with stronger hard X-ray flux in the IPC energy band. Thus radio-loud quasars are expected to have stronger Fe II(opt) emission, i.e., since they are both more luminous and have flatter slopes. This is opposite to the trend suggested by the observations and presents an interesting theoretical problem. Modification of current photoionization models to include more realistic X-ray continua for the two classes of quasars are essential to further understanding this situation.

It is also possible that these models are simply inadequate in their prediction of the Fe II strength. They have proved unable to reproduce the high observed Fe II strengths without invoking at least one additional component with lower ionization parameter and higher density (Collin-Souffrin, Dumont, and Tully 1982; Wills, Netzer, and Wills 1985; Joly 1987; Wills 1987). Successful modeling of the Fe II production may give a different prediction for the $\mathrm{X}$-ray/ $\mathrm{Fe} \mathrm{II}(\mathrm{opt})$ relation.

Other possible explanations of the $\mathrm{Fe} \mathrm{II}(\mathrm{opt}) /$ radioloudness relation have been suggested. For example, Wills and Browne (1986) and Miley and Miller (1979) have shown that compact radio quasars have significantly narrower emission lines than extended radio quasars. The more severe blending of the Fe II emission lines which occurs for quasars with broader emission lines hides the underlying continuum reducing the apparent $\mathrm{Fe}$ II strength and causing an apparent difference between its strength in compact and extended radio quasars (Wills, Netzer, and Wills 1985; Wills 1987). They emphasize that accurate measurements of Fe II emission-line strength can only be obtained via detailed modeling of the observed spectrum to take into account this line blending (Wills, Netzer, and Wills 1985). This procedure has not yet been applied to radio-quiet quasars in order to determine whether the radio-loud/radio-quiet $\mathrm{Fe} \mathrm{I}(\mathrm{opt})$ discrepancy could also be explained by this effect. However, radio-quiet and compact radio-loud quasars generally have similar line widths while the discrepancy between their Fe II(opt) strengths appears to be much more marked than for the two classes of radio-loud quasars. For example, the sample of Bergeron and Kunth (1984) shows no significant difference between the Fe II(opt) strengths for compact and extended radio-loud quasars while that for radio-loud and radio-quiet is very strong (see their Fig. 9). Thus, although confirmation must await more detailed study, it seems unlikely that line blending alone is the cause of the discrepancy (Osterbrock 1985).

In our small sample we cannot rule out the possibility that the line width is affecting our Fe II $\lambda 4570$ measurements. In Table 1 it is apparent that those quasars with narrow lines and strong $\mathrm{Fe}$ II are also those with steep X-ray slopes. The correlation between $\mathrm{Fe} \mathrm{II}(\mathrm{opt})$ and line width does not appear as strong as that with X-ray slope, but we cannot confirm this suspicion with our small dataset. If the line width is causing our $\mathrm{Fe} \mathrm{II}(\mathrm{opt}) / \alpha_{E}$ relation, then there must be a relation between line width and $\alpha_{E}$, which in turn must be explained.

Finally, physical mechanisms connecting $\mathrm{Fe}$ II and radio emission have been suggested by Ferland and Mushotzky (1984) and by Boyd and Ferland (1987). The former suggests that the relativistic gas which produces the synchrotron radio emission is mixed with the gas in BELR. The interaction of cosmic-ray electrons with the BELR gas heats the neutral gas sufficiently to quench the Fe II emission. The latter paper uses $\gamma$-rays to destroy the heavy elements, including $\mathrm{Fe}$ in the BELR gas. A Fe II/radio-loudness connection follows if 
radio-loud quasars are also $\gamma$-ray-loud, a logical extension of their X-ray loudness. Although both models predict the observed relation between optical Fe II(opt) and radio emission, they cannot explain the observed lack of a similar relation for UV Fe II (Bergeron and Kunth 1984).

\section{CONCLUSIONS}

Based on nine quasars we suggest that the soft X-ray slope is related to the $\mathrm{Fe} \mathrm{II}(\mathrm{opt})$ emission. The well-known statistical link between $\mathrm{Fe} \mathrm{II}(\mathrm{opt})$ and radio emission of quasars and active galactic nuclei is then secondary, a result of the strong link between the soft X-ray and radio emission. A link with the ionizing continuum seems physically more reasonable than one with the radio luminosity.

More generally, this result shows that soft X-ray spectra provide a new tool for understanding quasar emission lines and for testing photoionization models for the BELR. It is clear that careful measurements of the optical emission lines for individual quasars with well-determined $X$-ray spectra are essential to a systematic study. Since the known Fe II(opt)/radio loudness relation possesses a good deal of scatter, a large sample of radio-quiet and radio-loud quasars with X-ray slopes must be studied to understand these and any additional correlations fully. Should photoionization models prove unable to reproduce the observations, we will be forced to conclude that there is a more important difference between flat X-ray spectrum (radio-loud) and steep X-ray spectrum (radio-quiet) quasars which determines the relative strength of optical and UV Fe II emission.

We thank G. A. Kriss, M. Malkan, J. Bechtold, and R. F. Green for permission to use their data in advance of publication and B. M. Peterson for originally suggesting this possibility. Useful discussions with $\mathrm{H}$. Netzer, B. Wills, G. Ferland, and T. Kallman are also gratefully acknowledged. This work was supported in part by NASA contract NAS830751.

\section{REFERENCES}

Bechtold, J., Czerny, B., Elvis, M., Fabbiano, G., and Green, R. F. 1987, Ap. J., 314, 699 .

Bergeron, J., and Kunth, D. 1984, M.N.R.A.S., 207, 263.

Boyd, R. N., and Ferland, G. J. 1987, Ap. J. (Letters), 318, 21.

Collin-Souffrin, S., Dumont, S., Heidmann, N., and Joly, M. 1980, Astr. Ap., 83, 190.

Collin-Souffrin, S., Dumont, S., and Tully, J. 1982, Astr. Ap., 106, 362

Ferland, G. F., and Mushotzky, R. F. 1984, Ap. J., 286, 42.

Ferland, G. F., and Shields, G. A. 1985, in Astrophysics of Active Galaxies and Quasi-stellar Objects, ed. J. Miller (Mill Valley, Ca: University Science Books), p. 157.

Hutchings, J. B., and Gower, A. C. 1985, A.J., 90, 405

Joly, M. 1987 , Astr. Ap., in press.

Kazaryan, M. A., Carswell, R. F., and Khachikyan, E. E. 1974, Astr. Tsirk., 813, 2 .

Kembhavi, A., Feigelson, E. D., and Singh, K. P. 1985, M.N.R.A.S., 220, 51 .

Kriss, G. A., McHardy, I., and Malkan, M. 1987, in preparation.

Krolik, J. H. 1987 in Proc. of the GMU Conference Supermassive Black Holes ed. M. Kafatos (Cambridge: Cambridge University Press), in press.

Kwan, J., and Krolik, J. H. 1981, Ap. J., 250, 478.

Miley, G. K., and Miller, J. S. 1979, Ap. J. (Letters), 228, L55.

Netzer, H., and Wills, B. J. 1983, Ap. J., 275, 445.

Oke, J. B. 1974, Ap. J. Suppl., 27, 21.

Osterbrock, D. E. 1977, Ap. J., 215, 733.

1985, in Astrophysics of Active Galaxies and Quasi-stellar Objects, ed. J. Miller (Mill Valley, Ca.: University Science Books), p. 111.

Peterson, B. A., Wright, A. E., Jauncey, D. L., and Condon, J. J. 1979, Ap. J., 232, 400.

Peterson, B. M., Foltz, C. B., and Byard, P. L. 1981, Ap. J., 251, 4.

Phillips, M. M. 1977, Ap. J., 215, 746.

Remillard, R., and Schwartz, D. A. 1987a, Bull. AAS, Vol. 18, No. 4, p. 915.

Taylor, B. G., Andersen, R. D., Peacock, A., and Zobl, R. 1981, Space Sci. Rev. 30, 479

Wilkes, B. J. 1986, M.N.R.A.S., 218, 331

Wilkes, B. J., and Elvis, M. 1987, $A p$. $J$., in press.

Wilkes, B. J., Wright, A. E., Jauncey, D. L. and Peterson, B. A. 1983, Proc. Astr. Soc. Australia, $5,2$.

Wills, B. J. 1987, in IAU Colloquium 94, Physics of Formation of $\mathrm{Fe}$ II Lines outside LTE, ed. R. Viotti, A. Vittone, and M. Friedjung (Dordrecht: Reidel), in press.

Wills, B. J., and Browne, I. W. A. 1986, Ap. J., 302, 56

Wills, B. J., Netzer, H., Uomoto, A. K., and Wills, D. 1980, Ap. J., 237, 319 .

Wills, B. J., Netzer, H., and Wills, D. 1985, Ap. J., 288, 94.

Zamorani, G., et al. 1981, Ap. J., 245, 357.

MARTIN Elvis and Belinda Wilkes: Harvard-Smithsonian Center for Astrophysics, 60 Garden Street, Cambridge, MA 02138

IAN MCHARDY: X-Ray Astronomy Group, University of Leicester, Leicester LE1 7RH, England 